\title{
PRELIMINARY EXPERIMENTAL/NUMERICAL STUDY OF THE VIBRATION ANNOYANCE CONTROL OF A WINDSHIELD WIPER MECHANICAL SYSTEM THROUGH A SYNCHRONIZED SWITCH SHUNT RESONATOR (SSSR) TECHNOLOGY
}

\author{
Massimo Viscardi, Romeo Di Leo \\ University of Naples "Federico II", Department of Industrial Engineering - Aerospace Section, Naples, Italy \\ e-mail: romeodileo@gmail.com \\ Monica Ciminello \\ C.I.R.A. Italian Aerospace Research Centre, Capua (CE), Italy \\ MARCO BRANDIZZI \\ C.R.F. Centro Ricerche Fiat, Orbassano (TO), Italia
}

\begin{abstract}
The present work focuses on the study and analysis of vibrations generated by the electrical motor of a wiper system on its support bracket referring to an hatchback vehicle passenger car. In the electric motor of the wiper system there can be present an imbalance transmitting vibrations to the main body via the support bracket. In this paper, after a short resume of available synchronized switch shunt damping methods, a preliminary experimental evaluation of their potential performance on noise control of the wiper system is reported. After preliminary experimental measurements of dynamic vibration by the use of vibrometer laser both on the real hatchback car then in laboratory environment, a numerical model has been created to evaluate deformations of the support bracket for comparison with the experimental data. This work realizes the preliminary numerical/experimental characterization activity to set up a new application of a control system based on a semi-active technique, called Synchronized Switch Shunt Resonator (SSSR).
\end{abstract}

Keywords: vibration control, acoustic, wiper system, semi-active technique, synchronized switch shunt resonator

\section{Introduction}

In the modern automotive industry, the fundamental topic is noise annoyance inside the car.

The reduction of internal noise is of great importance in order to make the driving experience safer and more comfortable and to prevent that these annoyance sources could be perceived as the low quality indicator. For this reason, great efforts are addressed by the research world to define requirements and specifications for integrated solutions aimed at reduction of noise using different cost effective established technologies such as noise and vibration control, innovative materials and optimized tires, analyzing related risks too. In addition, many new soundproofing solutions are developed and focused, in particular, oriented on internal noises reduction (especially for squeak and rattle noises).

The noise sources in an auto-vehicle can be divided into the primary and secondary sources. In the first group, there are three main sources:

- The power-train: the engine noise is generated by mechanical and chemical processes inside the engine that cause vibrations which are transmitted to the cockpit.

- Interaction road-tires: the road noise is generated by the interaction with the tire interface and the ground and causes broadband vibrations which are transmitted to the structure. 
- Aerodynamic flow: the vortex shedding noise which is caused by the fluid (air) and a non-aerodynamic body (car) interaction.

In the other group, there are two main secondary sources:

- Noise generated internally by the passenger compartment (squeaks, etc.).

- Equipment and systems of various kinds (air conditioning, etc.).

Within this frame, the present work focuses on the study and analysis of vibrations generated by the electrical motor of the wiper system transmitted on its support bracket for a hatchback vehicle passenger car application. It has been indeed identified that the electric motor of the wiper system can present an imbalance transmitted to the body through this support bracket. The main goal is to investigate typical vibration modes of the supporting plate in order to design proper control system parameters based on a semi-active technique. This technique uses piezo transducers to convert mechanical into electrical energy and elaborates the related signal within an external electrical circuit.

Piezoelectric materials fall in the category of the so-called smart materials and they can often control and suppress vibration in an efficient and intelligent way without causing much additional weight or cost. In many research activities, piezoelectric materials are satisfactorily applied to control structural materials due to their excellent mechanical-electrical coupling characteristics.

The shunt methods for vibro-acoustic control based on piezoelectric sensors and actuators can be divided into three main classes: passive, active and semi-active. Passive shunt control systems mainly reproduce the dynamic vibration absorber device by means of electrical components, typically resistive-inductive (R-L) circuits (Hollkamp, 1994). This kind of circuits is simple to design but its performance is based on the resonance tuning, so it is sensitive to variations of structural parameters. In addition, R-L shunt systems need large inductance for a low frequency domain, which is improbable in realistic applications.

Active control systems require high-performance digital signal processors and bulky power amplifiers to drive actuators, which is not suitable in many practical applications too. To overcome these disadvantages, several semi-active approaches have been proposed. In the last years, Clark proposed a state-switched method (Clark, 1999) in which piezo-elements were periodically held in an open-circuit state, then switched and held in the short-circuit state synchronously with the structure motion. Another type of semi-active control, which has been receiving much attention in the recent years, is called the pulse switching technique (Richard et al., 1998; Onoda et al., 2003; Makihara et al., 2005). It consists in fast inversion of voltage on the piezo-element using a few basic electronic elements synchronized with the mechanical vibration.

In the methods proposed by Richard et al. (1998), the voltage is switched on the piezoelectric element at each strain extrema or, equally, displacement extrema. These methods are called synchronized switch damping (SSD) techniques (Ciminello et al., 2008).

The present paper reports a preliminary feasibility study of the well established control technology on a new automotive application. The Synchronized Switch Shunt Resonator (SSSR) control system already investigated and applied by the authors for aeronautical components (Ciminello et al., 2010; Ameduri and Ciminello, 2010) is used here to a new particular test case, namely a wiper mechanism for an automotive application. The paper at hand includes a preliminary experimental evaluation of the control of the wiper assembly vibrations supported by some FEA computations. The SSSR design is also described and followed by preliminary experimental results. The authors show possible issues with realistic damping of low amplitude vibrations of the wiper system bracket. 


\section{Synchronized switch damping (SSD) method}

The synchronized switch damping (SSD) method, also called the pulse-switched method, consists of nonlinear processing of the voltage on a piezoelectric actuator. It is implemented with a simple electronic switch synchronously driven with the structural motion.

This switch, which is used to reverse the voltage on the piezoelectric element, allows one to briefly connect a simple electrical network (short circuit, inductor and voltage sources depending on the SSD version) to the piezoelectric element. Due to this process, a voltage magnification is obtained and a phase shift appears on the resulting voltage. The force generated by the resulting voltage is hence opposite to the structural motion, thus creating energy dissipation. The dissipated energy corresponds to the part of the mechanical energy converted into electric energy. Maximizing this energy is equivalent to minimizing mechanical energy in the structure.

Several SSD techniques are reported. The simplest is called SSDS, as shown in Fig. 1, which stands for synchronized switch damping on the short circuit (Clark, 1999).

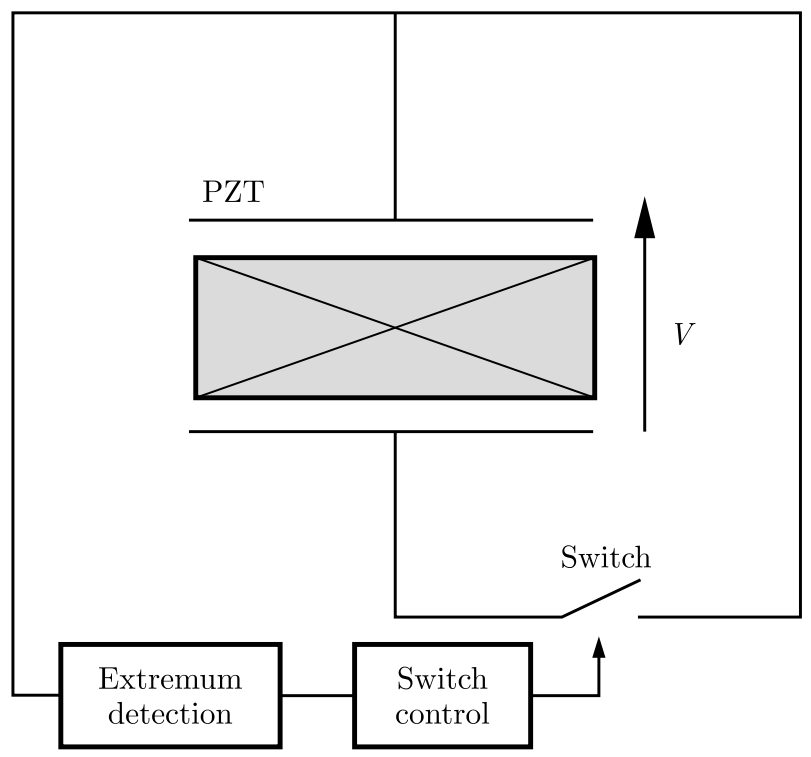

Fig. 1. Principle of the SSDS technique

The SSDS technique consists of a simple switching device in parallel with the piezoelectric patch without other electric devices. The switch is kept open for most of the time during the vibration period and then closed when the voltage reaches the maximum (corresponding to the maximum of the strain on the piezoelectric patch) and left closed for a short period in order to dissipate the stored electric energy.

According to the work of Qiu et al. (2009), for the SSDS circuit, the transferred energy $E_{t}$ in a period is given by

$$
E_{t}=\frac{4 \alpha^{2}}{C_{0}} u_{M}^{2}
$$

where $\alpha$ is the piezoelectric coefficient, $C_{0}$ is the capacitance of the piezoelectric element and $u_{M}$ is the amplitude of vibration (Badel et al., 2006).

To further increase the dissipated energy, the SSDI technique (synchronized switch damping on the inductor) shown in Fig. 2, has been developed by Richard et al. (1998), Guyomar et al. (2001) and Petit et al. (2004).

In the SSDI approach, the inductor is connected in series with the switch. Because the piezoelectric patch and the inductor constitute an L-C resonance circuit, fast inversion of the 
voltage on the piezoelectric patch is achieved by appropriately controlling the closing time and duration of the switch.

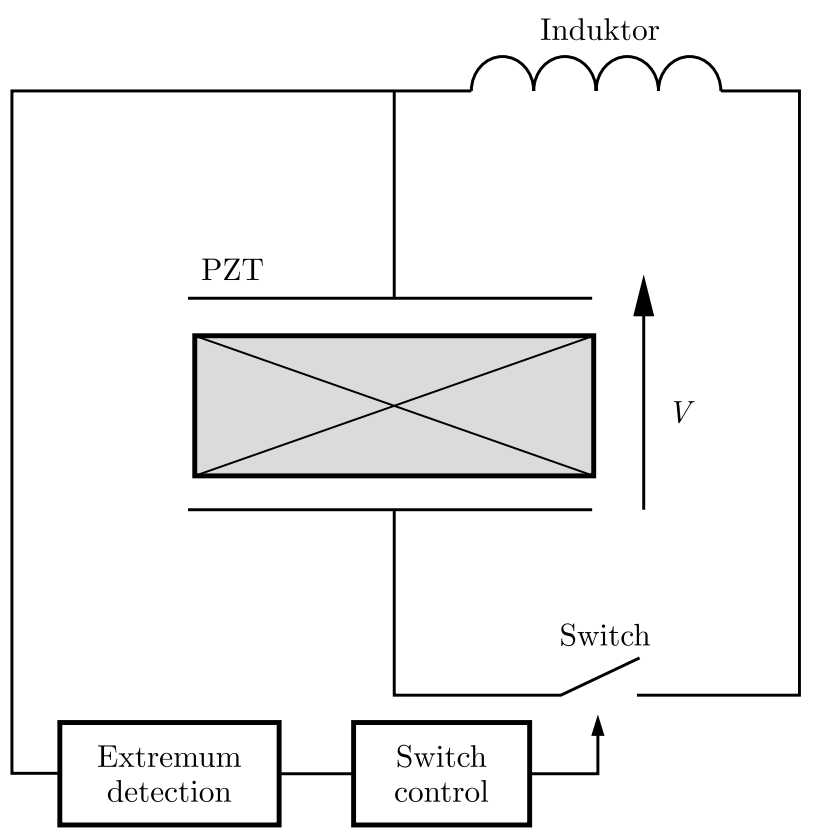

Fig. 2. Principle of the SSDI technique

The switch is closed at the displacement extremes, and the duration of the closed state lasts half of the period. This leads to an artificial increase of the dissipated energy. The period of the $\mathrm{L}-\mathrm{C}$ circuit is chosen to be much smaller than that of the mechanical vibration.

In the SSDI technique, the dissipated energy $E_{t}$ during the period is given by

$$
E_{t}=\frac{4 \alpha^{2}}{C_{0}} \frac{1+\gamma}{1-\gamma} u_{M}^{2}
$$

where $\gamma \in[0,1]$ is the voltage inversion coefficient. Comparing equations (2.1) and (2.22), the transferred energy is magnified by means of $(1+\gamma) /(1-\gamma)$ factor (Badel et al. (2006).

Although most semi-active studies have been devoted so far to single-mode control, the switching laws can be also used for multi-mode control. In the method proposed by Makihara et al. $(2007 \mathrm{a}, \mathrm{c})$, switch actions are automatically generated by the control law applicable to multi-modal control of a structure with multiple piezoelectric transducers.

In the method proposed by Ciminello et al. (2010), a similar technology was tested to verify its experimental performance on a $2 \mathrm{D}$ structure over a broadband frequency range. The selected test specimen was a $220 \mathrm{~mm} \times 280 \mathrm{~mm}, 7 \mathrm{~mm}$ thick, 10-ply fiberglass laminate plate. The system was made of several actuator/sensor piezoelectric ceramic pairs linked to a 4-channel control circuit. Active elements were embedded into the structure at different locations to produce effects over a large number of modes. The placement resulted from an optimization study performed to maximize their action over the frequency bandwidth of interest. The equivalent induced damping was estimated for seven out of eight bending modes present in the investigated interval. Amplitude reductions up to $16 \mathrm{~dB}$ were attained. The experimental results were found to be in good accordance with the numerical predictions.

Through about 10 years of research, several electronic switching circuits and switch control laws have been developed. However, more improvements must still to be provided for practical applications. Due to the energy loss during voltage inversion, these switching laws are not always optimal as demonstrated by experimental results (Makihara et al., 2007b; Viscardi and Leo, 2016). More efficient control laws and higher robustness of the control system are required. 


\section{Problem formulation}

The target of the activity at hand is the vibration control of the mechanical wiper system of a hatchback passenger car. The system is linked to the chassis of the vehicle in three points (red circles in Fig. 3).

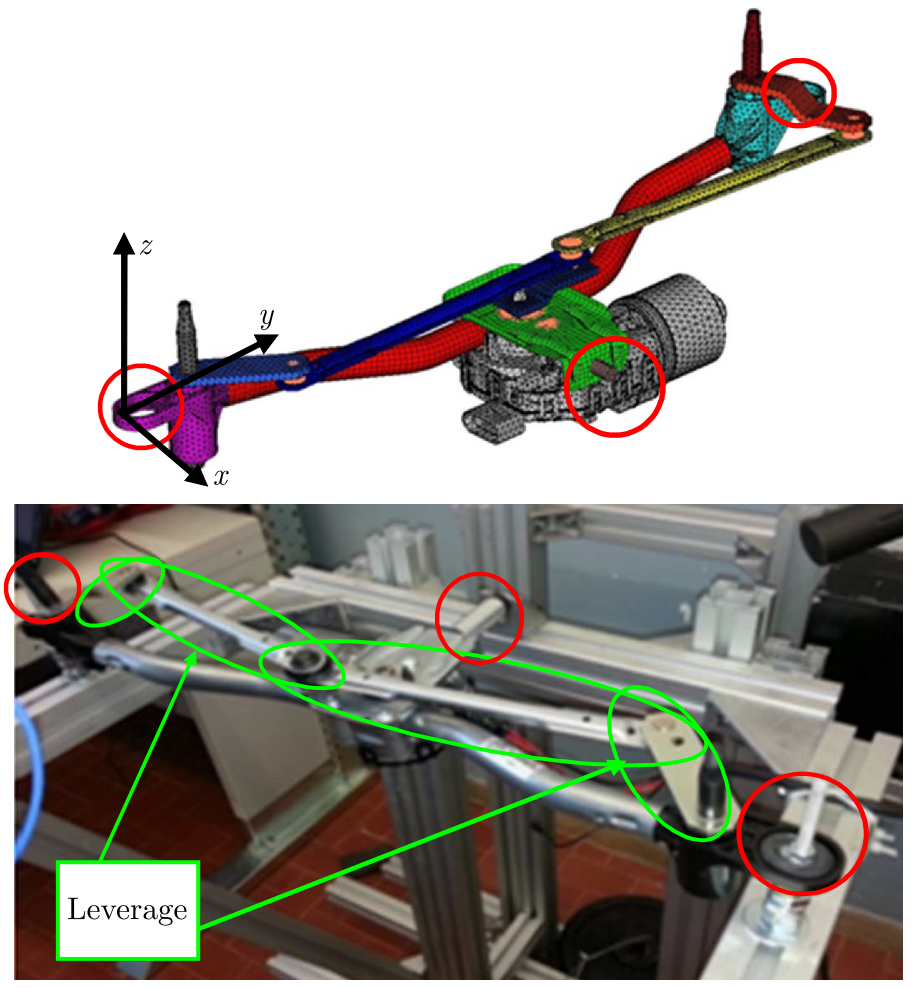

Fig. 3. Digital and physical mock-up of the wiper system

It has been identified that the electric motor of the wiper system presents an imbalance transmitting vibrations to the main body via the support bracket (part in red colour in Fig. 4).

According to this problem and to the specifications (that is the reduction of vibrations in the frequency range $600-850 \mathrm{~Hz}$, which is the range of interest as provided by the experimental measures on the car and as said in the subsequent lines), a feasibility study on the vibration suppression provided by a semi-active synchronized switch shunt resonator (SSSR) is investigated.

Specific analysis with the wiper system of the car used as the test case is addressed to measure the force transmitted to the chassis of the car through the constraint points of the wiper generated by the electrical motor in operating conditions. Figure 5 shows the force spectrogram transmitted in the $Z$ direction according to the reference system in Fig. 3. The force is measured on the right lateral constraint and shows a maximum peak in the $600-850 \mathrm{~Hz}$ frequency range. The red curve is the measured force with the wiper blades mounted, and the blue curve refers to the wiper blades dismissed.

The design and manufacturing of the dedicated control system has been developed through the next steps:

- Measurements of the acoustic performance for the original wiper system (without system control) in the real hatchback passenger car cabin.

- Dynamic characterization of the support bracket in the wiper system.

- Numerical simulation of the dynamic behaviour of the support bracket in the wiper system.

- Design of the basic layout of the control system. 


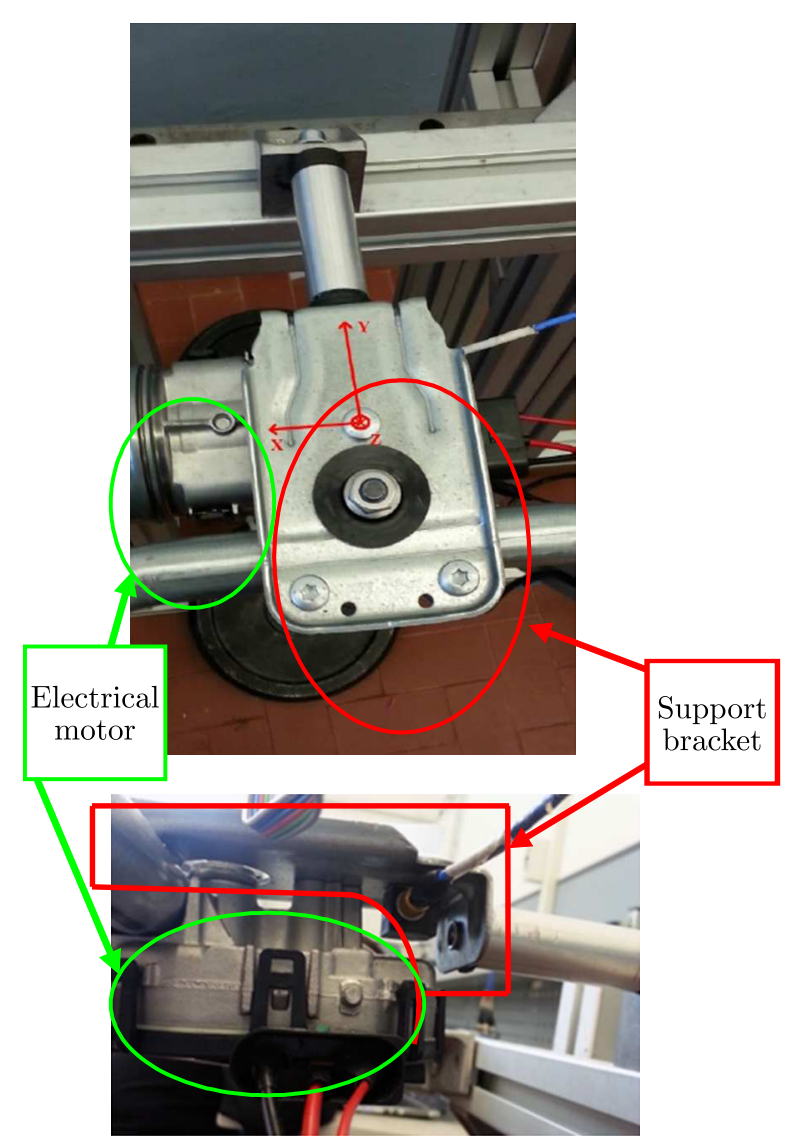

Fig. 4. Assembly bracket with an electrical motor. The upper and lateral view

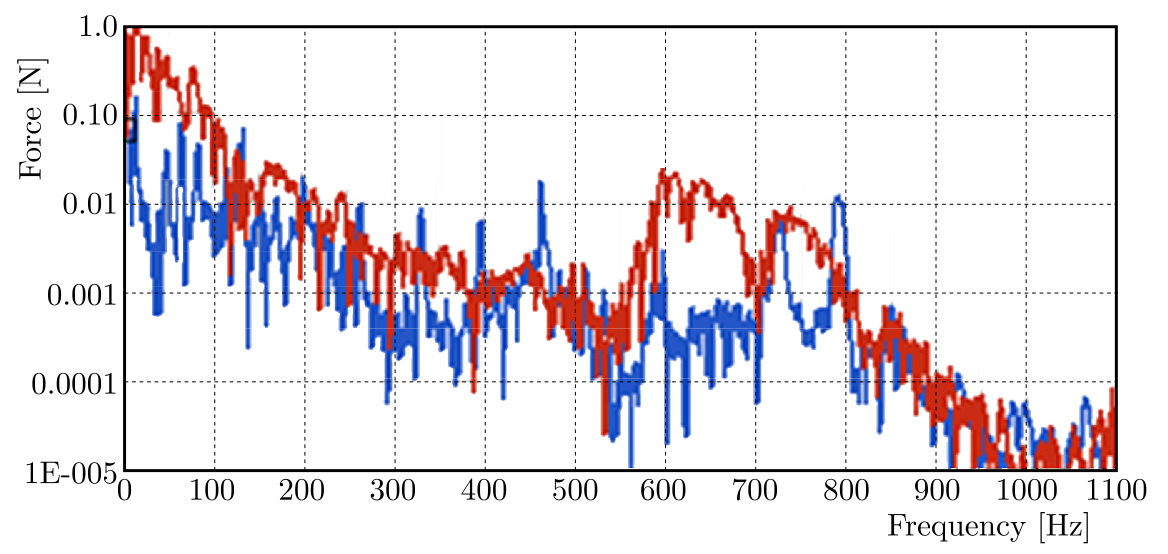

Fig. 5. Force wiper system-chassis in the $Z$ direction on the right lateral contraint

\section{Acoustic performance of the wiper in the vehicle cab}

The acoustic performance of the mechanical wiper has been measured. Measurements were performed by the authors in different operative speeds of the wiper system and with thermodynamic engine in the "off" and "on" state.

The experimental set-up was composed by two Roga MI-17 Icp microphones which were installed on both sides of the head of the driver at the ear level. An additional Roga MI-17 Icp microphone was installed outside the vehicle in front to the bonnet at a distance of one meter.

Besides, an accelerometer was installed on the bottom face of the support bracket to measure the vibrating level produced by the electrical motor in real operative conditions. Another one 
was positioned on the interior surface of the windshield to measure acceleration transmitted by the wiper to have an idea about the most important transmission path between the windshield and the chassis.

For data acquisition, an LMS Scadas Mobile 5 with eight channels and TestLab software were used.

The first data logging was done for the accelerometers in two different conditions. The first one was characterized by the thermodynamic engine "on" at different rotational speeds (idle, $1500,2000 \mathrm{rpm}$ ) while keeping the wiper system "off". The second data logging was characterized by the thermodynamic engine "off" while keeping the wiper system "on". For every measure the car was left with the gearbox in neutral position.

Figure 6 shows that the acceleration of the support bracket produced by the excitation of the wiper electrical motor is higher than the one produced by the stand alone thermal engine. Therefore, the effect of the wiper on the vibrating energy, transmitted by the support bracket to the chassis is not negligible.

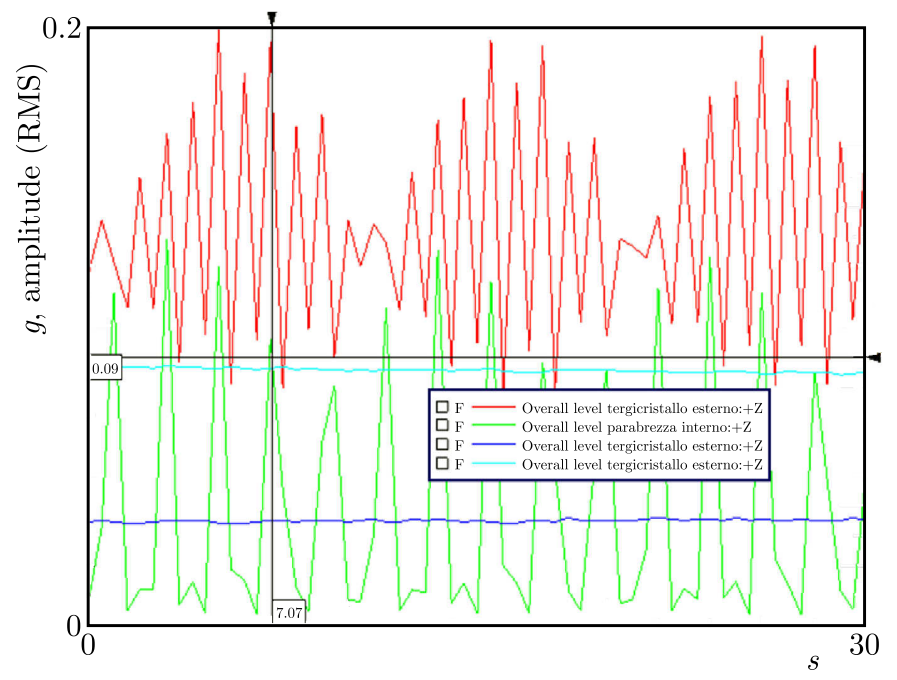

Fig. 6. Acceleration of the support bracket for the thermal engine "on" and the wiper "off" (blue curve - idle, cyan - $1500 \mathrm{rpm}$ ); acceleration of the support bracket (red curve) and the windshield (green curve) for the thermal engine "off" and the wiper "on"

Besides, the amplitude of acceleration of the support bracket, expressed in "g" units, is higher than the windshield one.

In the next figures, the background noise of the environment inside and outside the car are reported (the thermal motor and the wiper system in "off" condition) and besides the internal and external noise in the cases of three different wiper speeds (Vel1, Vel2 and Vel3).

Inside and outside the passenger compartment, the background noise is almost the same, even if at low frequencies is smaller inside due to attenuation generated by the windscreen of the car.

It can be noted that the noise with the wiper electrical motor "on" is higher than the background noise. The external noise is approximately the same for three different speeds of the wiper (Fig. 8), although the internal noise increases with the increasing of the speed (Fig. 9). 


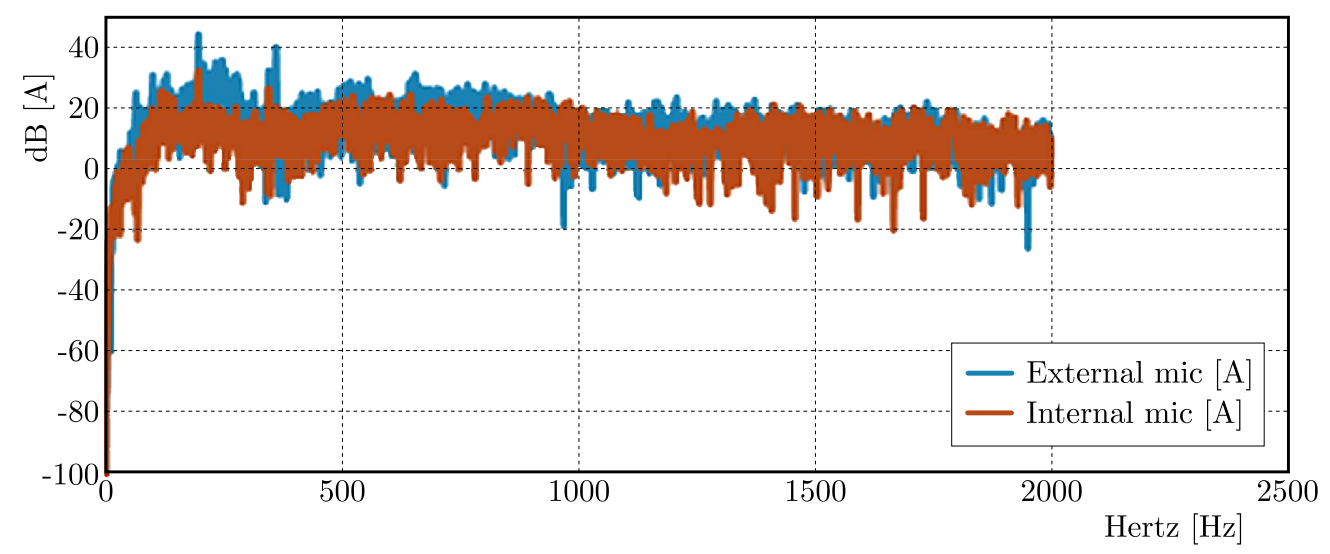

Fig. 7. Internal and external background noise diagrams $[\mathrm{dB}(\mathrm{A})]$

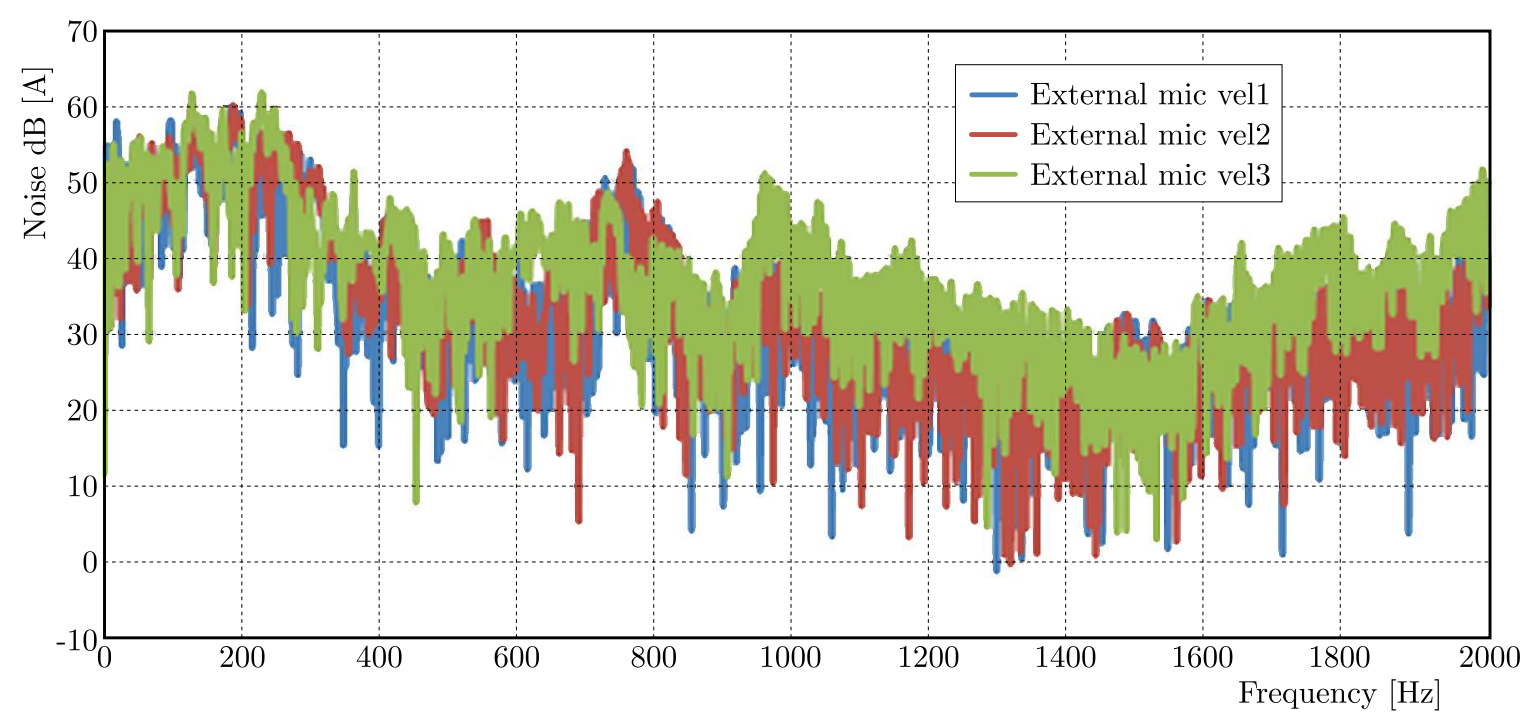

Fig. 8. External noise vs. variation of the wiper speed

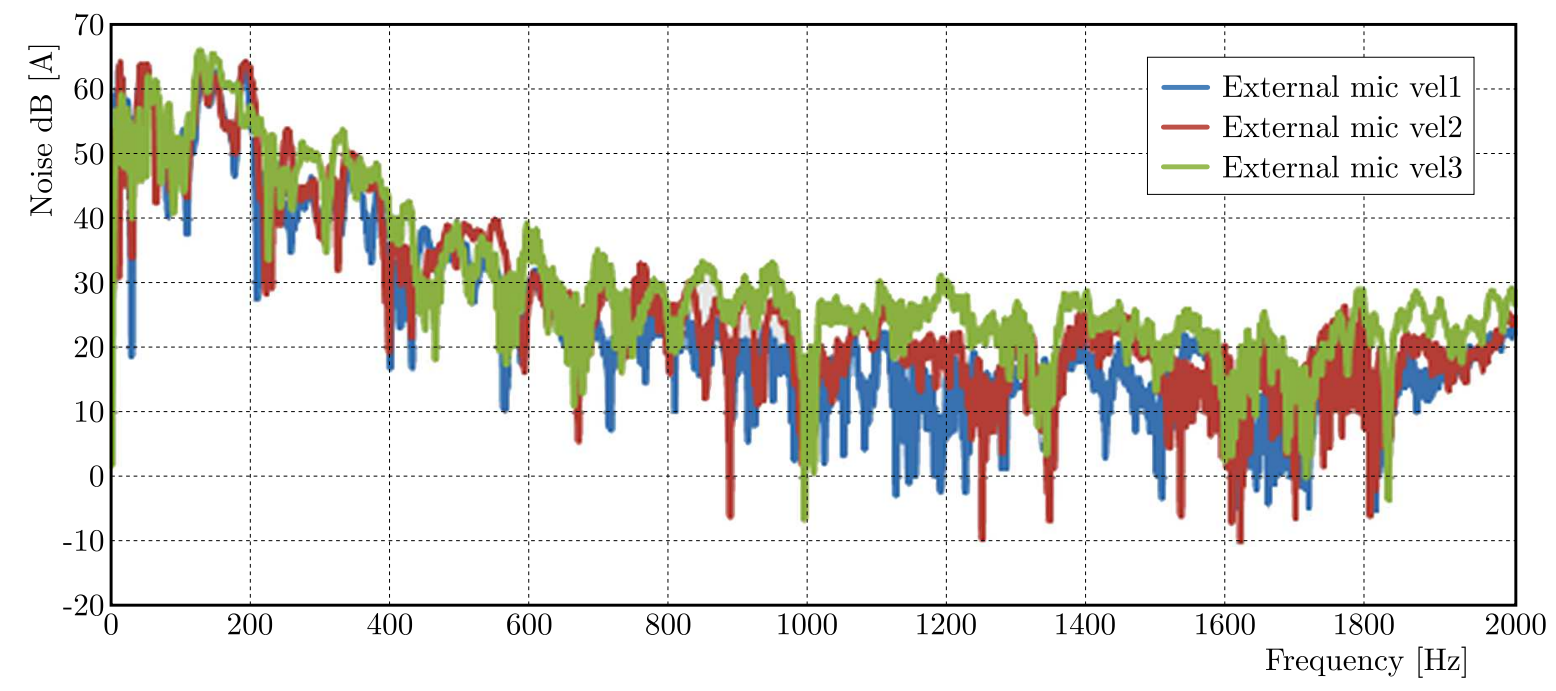

Fig. 9. Internal noise vs. variation of the wiper speed 
Besides, it is evident that the perceived noise outside the vehicle is always greater than $1 \mathrm{~dB}$ compared to that received inside. This is due to the windscreen that acts as a soundproof.

The tests performed with accelerometers and microphones confirm that there are high peaks of acceleration and pressure in the frequency range between $600 \mathrm{~Hz}$ and $850 \mathrm{~Hz}$ (blue circle in Fig. 10) validating the preliminary numerical FE analysis.

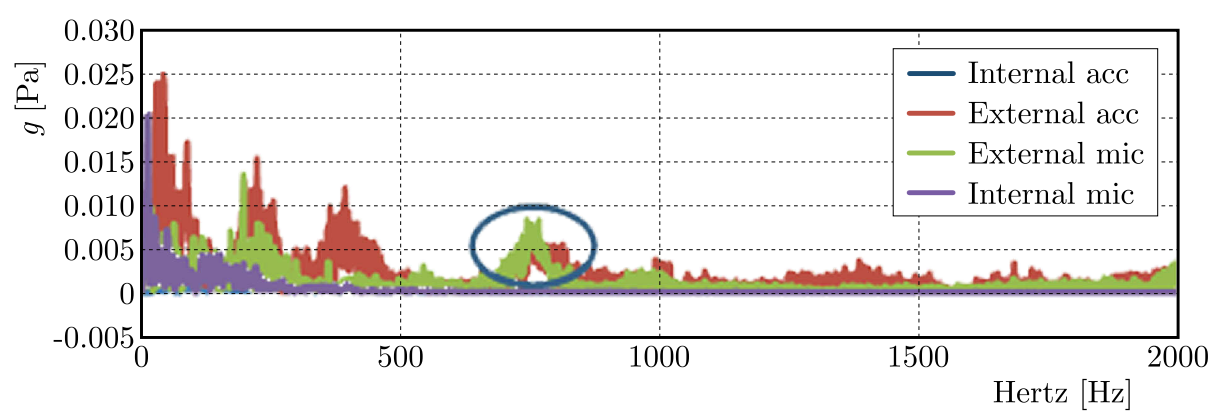

Fig. 10. Summary diagram of tests performed on the wiper at Vel2

\section{Experimental set-up and dynamic characterization}

In order to reproduce the same constraints with the chassis in the bonnet compartment of the car, an aluminum frame has been built carrying three clamping points. The wiper was installed on this frame (Fig. 3) in order to perform the test campaign.

At first, a dynamic characterization test was performed through the vibrations measured by a triaxial piezoelectric accelerometer placed on the bottom face of the support bracket (Fig. 4). The tests were carried out at two different electric motor velocities: Vel1 and Vel2. Moreover, the tests were conducted both with and without leverage (see Fig. 3) in order to left the support bracket free from the rotating kinematic system and to make vibrometer laser analysis (Fig. 11).

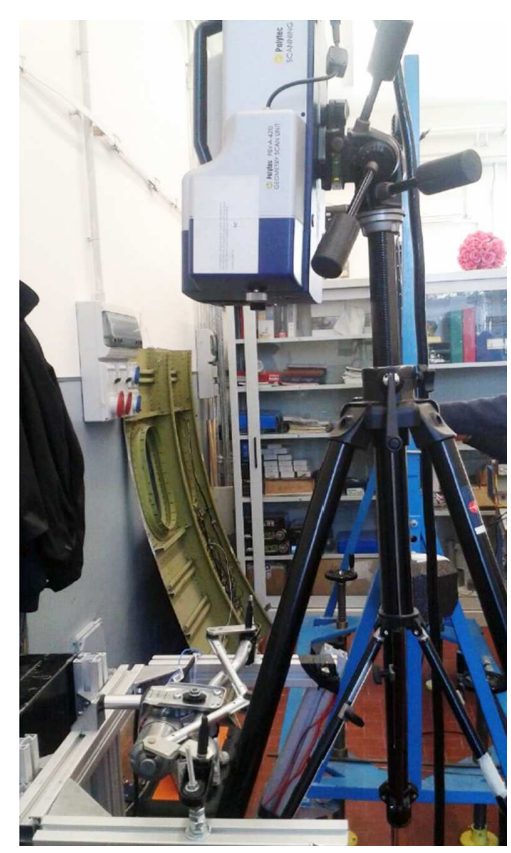

Fig. 11. Mechanical wiper system installed in the laboratory set-up with Polytec PSV-400 Scanning Vibrometer

Following graphs shows that the peak of acceleration has a light shift in the case of absence of leverage for both speeds of the wiper motor (from a value of $494 \mathrm{~Hz}$ to $540 \mathrm{~Hz}$ for Vel1 and from $760 \mathrm{~Hz}$ to $830 \mathrm{~Hz}$ for Vel2). 
Besides, from the data analysis it results that acceleration values measured without leverage are generally greater than those measured with the leverage; the only exception is the acceleration referred to the $y$ axis, which presents higher values when the leverage is present.

It is however clear that there is an influence of the presence of the leverage on the global dynamic behaviour of the wiper.

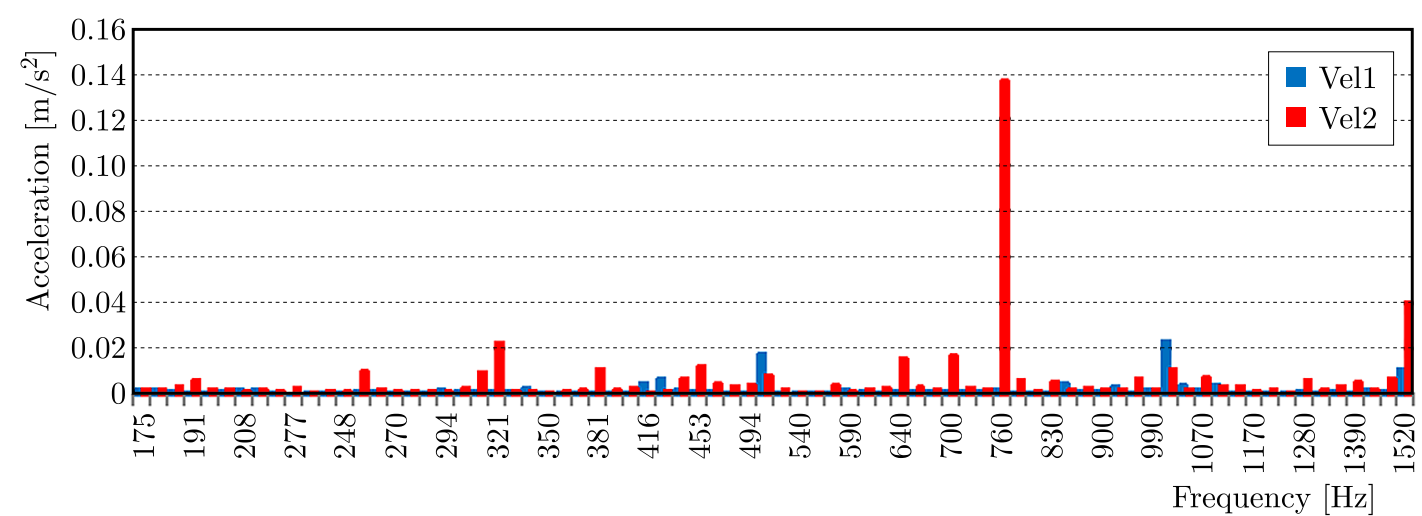

Fig. 12. Frequency spectrum ( $X$ axis) $1 / 24$ octave with leverage

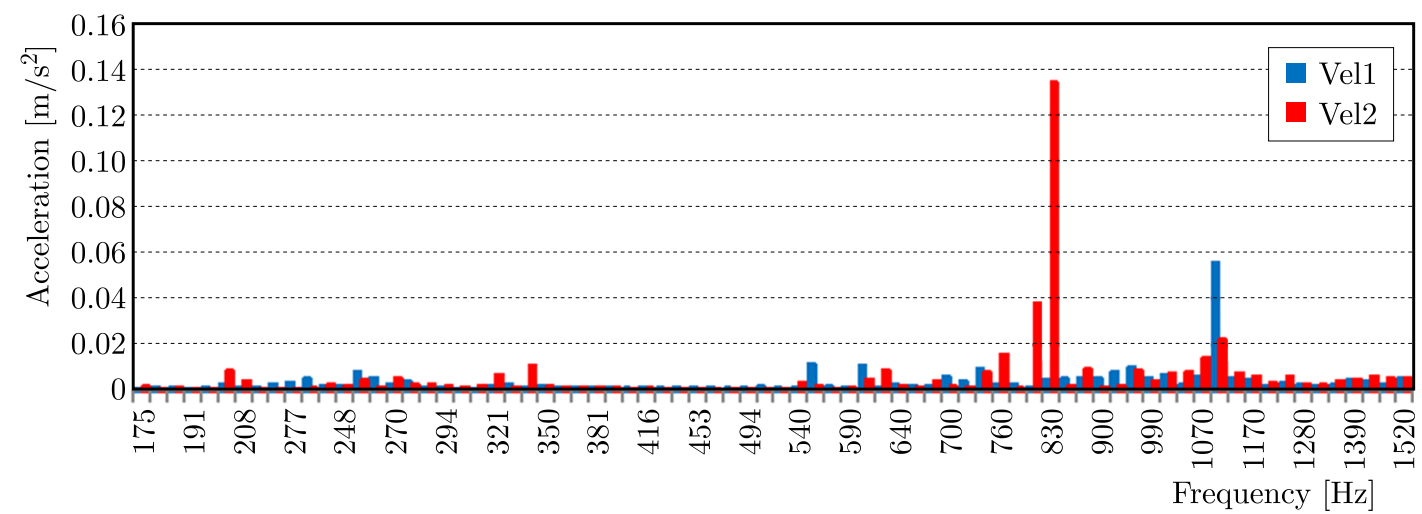

Fig. 13. Frequency spectrum ( $X$ axis) $1 / 24$ octave without leverage

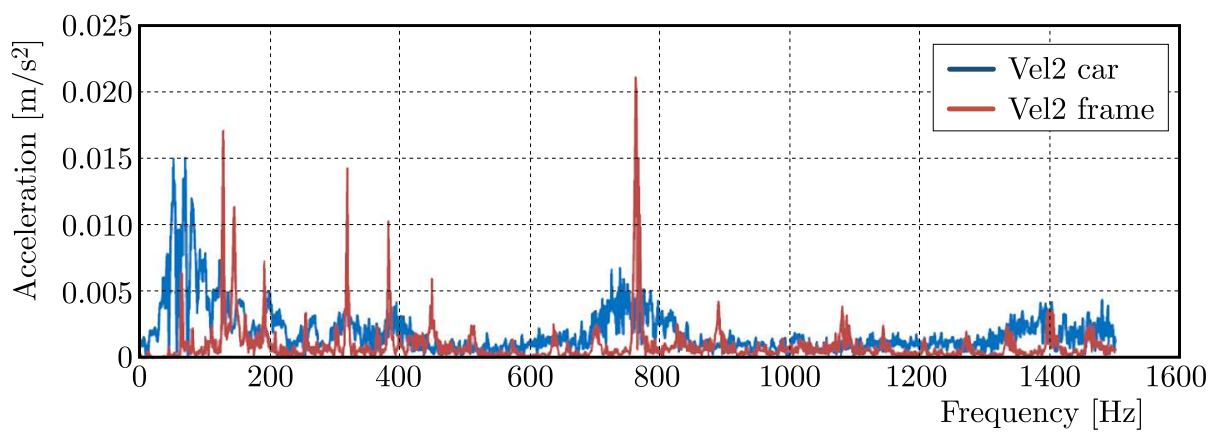

Fig. 14. Acceleration in the time domain ( $X$ axis) for tests on the car and on the set-up

There are some differences in the system behaviour depending on the typology of the main support (car chassis or frame structure).

In particular, the wiper blades provide a considerable amount of friction on the glass, which is an important noise source. To characterize the structural vibration of the support bracket, a Polytec PSV-400 scanning vibrometer was used. The laser was positioned at $550 \mathrm{~mm}$ from the surface of the bracket. In order to properly acquire displacements, velocities and accelerations of the brackets, all the following tests were carried out without leverage of the kinematic system because of their interference with the track of the laser beam. 
The measurements have been conducted tracing a scanning grid composed of 176 dots on the support bracket surface and detecting, through the scanning vibrometer, the velocity of each point. The test has been performed both at Vel1 and Vel2 in order to identify eventual differential behaviour, focusing on modal shapes and frequency resonances in the range between $500 \mathrm{~Hz}$ and $850 \mathrm{~Hz}$.

The following images (Figs. 15 and 16) have been achieved considering the Vel1 electric motor velocity.

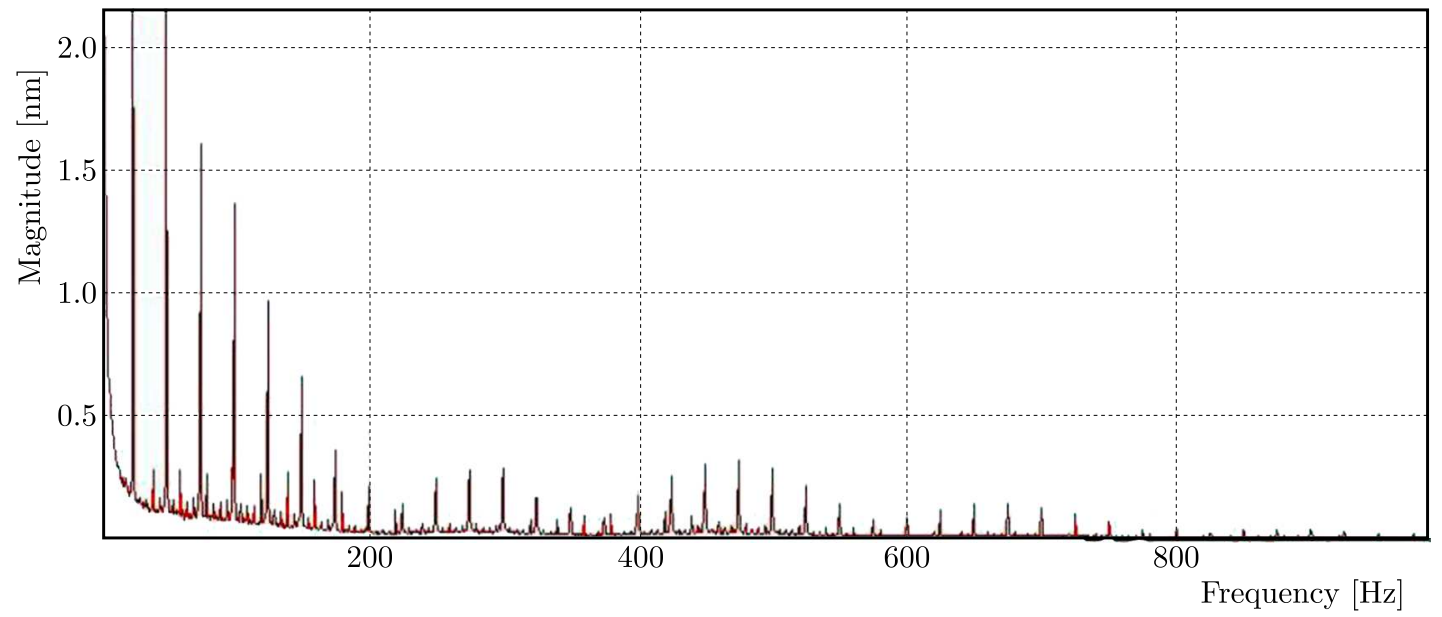

Fig. 15. FRF displacement vs frequency at Vel1 for the point of maximum displacement
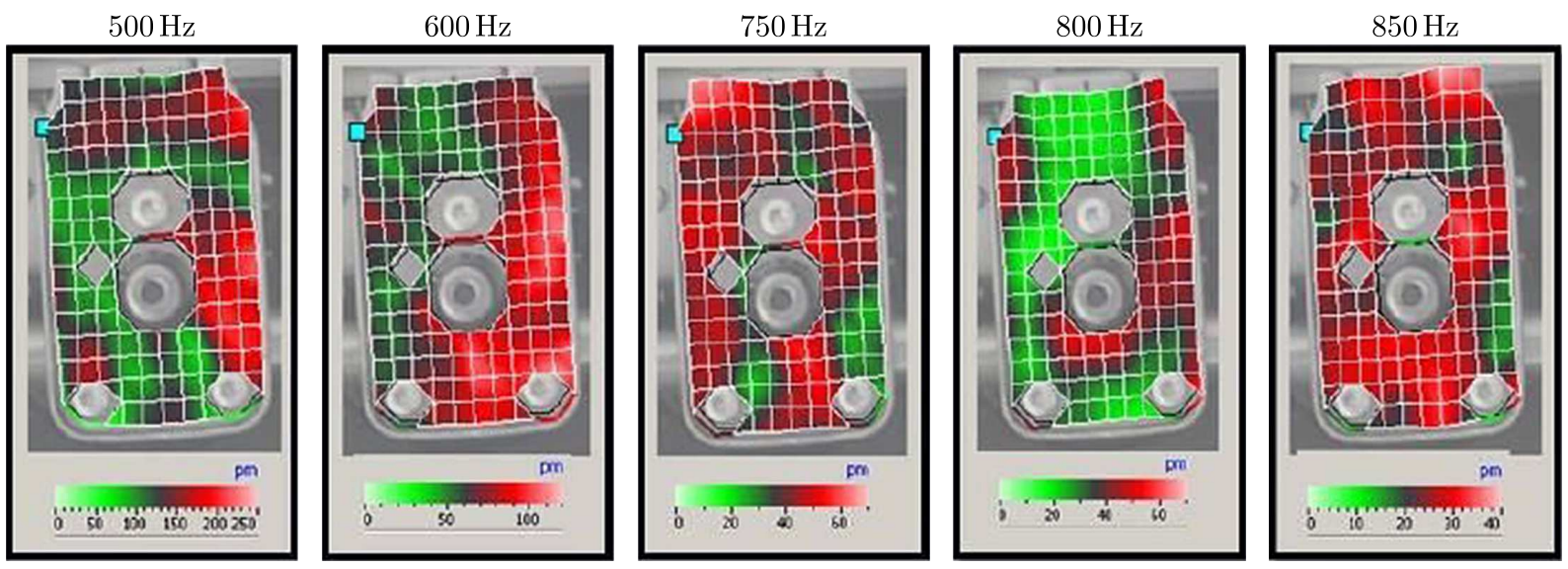

Fig. 16. Modes of vibrations images computed by PSV-400 scanning vibrometer at Vel1

It is clear that this curve is characterised by periodic behaviour with three repeated distinctive groups of peaks. According to this spectrogram, $500 \mathrm{~Hz}, 600 \mathrm{~Hz}, 750 \mathrm{~Hz}, 800 \mathrm{~Hz}$ and $850 \mathrm{~Hz}$ have been analysed in detail.

Modal shapes and strain distribution are required to optimize strain energy transmission from the SSSR to the bracket. It is in fact necessary to know the locations of the plate presenting the highest deformations in the frequency range during the test finalized to a good positioning of the piezo patch. The piezo devices must maximize the energy extracted from the mechanical part and convert it into the electrical energy for the control supply.

The map of displacements reported in Fig. 16 is used to compute the associated deformations by applying the following expression which comes from the definition of the discrete form of the second derivative linked to the strain by a half of the thickness $t$ for a small displacement

$$
\varepsilon=\frac{t}{2} \frac{w_{i-1}-2 w_{i}+w_{i+1}}{\Delta x^{2}}
$$


where $w$ is the transverse displacement measured by the vibrometer laser, and $x$ is the coordinate in the plane.

In reference to the transversal displacements of the plate for the support bracket at $750 \mathrm{~Hz}$, the maximum deformation is $4.4 \cdot 10^{-4} \mu \varepsilon$.

\section{Numerical analysis}

The Nastran SOL111, has been used to verify the map of displacements at $750 \mathrm{~Hz}$. In particular, the analysis shows that the modal shape for this frequency is characterized by a torsional deformation of the bracket around the axis of the central rivet connection (Fig. 17), thus highlighting the region with highest deformations and in accordance with the experimental data (blue circle in Fig. 16).
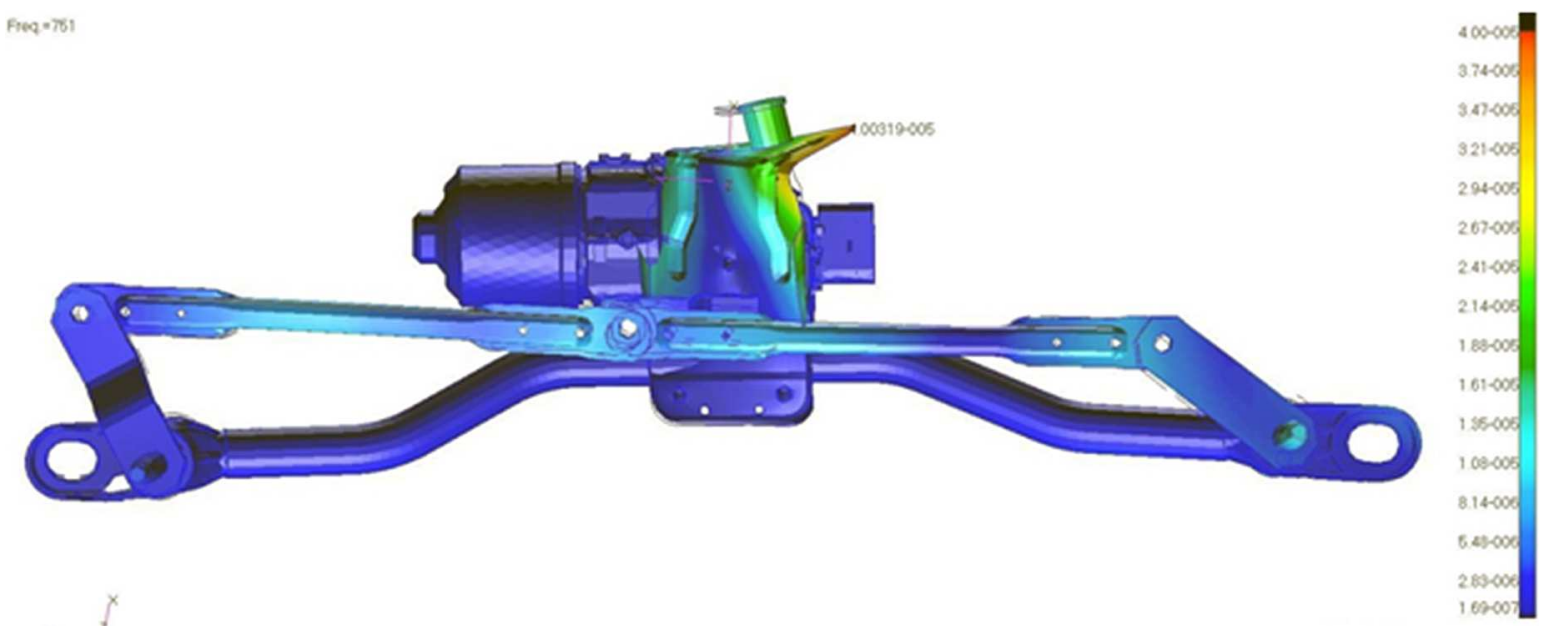

Fig. 17. Modes of vibration at $750 \mathrm{~Hz}$ by FEM analysis

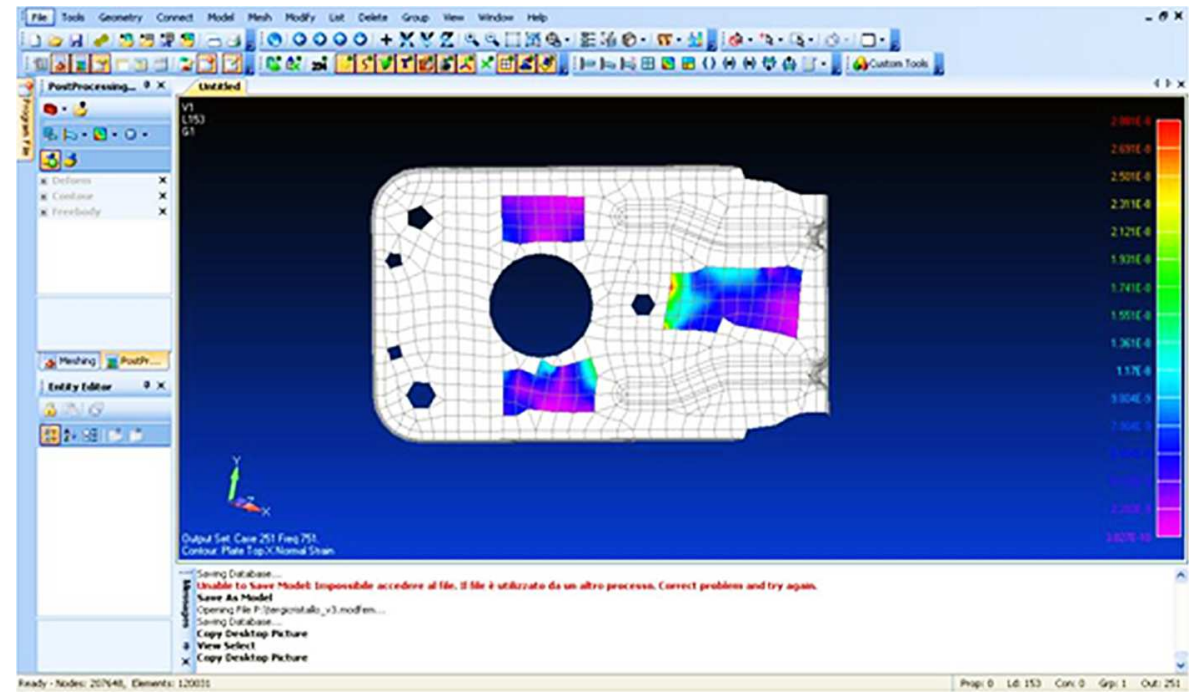

Fig. 18. In plane strain map

The strain energy map is also plotted, which shows that in the plane of the bracket the areas of most energy are around the central rivet and holes, as expected (Fig. 18). This analysis highlighted the areas of interest for deformation energy congruent with the experimental data. 
In addition, this area is a unique suitable area, flat enough to bond the piezoelectric patch. In the same region, the finite element analysis shows deformation of the order of magnitude of $5 \cdot 10^{-4} \mu \varepsilon$ in accordance with the experimental valuation.

\section{Conclusion and future developments}

An original study of a windshield wiper mechanical system has been herein reported. Theoretical analysis highlighted a mechanical imbalance transmitting vibrations to the wiper main body via the support bracket. This problem can be of high interest for future electrical cars design, in which motor noise Siano et al. (2016) will be negligible with respect to other mechanical subsystems causing annoyance to passengers.

The paper reports a preliminary feasibility study of a well established control technology on a new automotive application. A semi-passive shunt control technique opportunely designed for the vibrations reduction within the required frequency band has been here used to a new particular test case, namely a wiper mechanism for an automotive application. The paper at hand includes a preliminary experimental evaluation of the wiper assembly for vibrations control, supported by some FEA computations. The SSSR design is also described, followed by preliminary experimental results. The authors show possible issues with realistic damping of low amplitude vibrations of the wiper system bracket. However this technique seems to more adequately face this type of application in respect to other ANC and AVC approaches (Magliacano et al., 2016a,b).

A real windshield wiper mechanical system has been then installed on a steel truss support. The constraint has been reproduced according to the car schematics, the engine has been powered and the transmitted vibrations have been detected via vibrometer laser instrumentation.

Due to complex geometry of the wiper characterized by sharp curvatures and local stiffeners, only short areas have been available for secondary bonding of sensors. Strain gauges and pzt have been installed and the energy strain level has been correlated and compared to the vibrometer output.

The results were in good agreement, thus showing the robustness of the predicted numerical evaluations and applied methodologies. Nevertheless, the results showed very low deformations. The implementation of a semi-passive shunt control could be applied with some restrictions. A Synchronized Switch Shunt Resonator proved to be the most promising technical solution, according to the mechanical specifications, but requiring a very sensitive transducer device on the same time.

\section{Acknowledgments}

The present work has been part of the Low Noise project, co-financed by the Italian University and Research Industry. The authors of the present work address an acknowledgement to all partners involved in the project but special thanks are addressed to Gaetano Arena for his strong, enthusiastic and effective support in the development of the experimental activity in the laboratory of Aerospace Section of the Industrial Engineering Department of the University Federico II in Naples.

\section{References}

1. Ameduri S., Ciminello M., 2010, Fourier expansion solution for a Switched Shunt Control applied to a duct, Journal of Theoretical and Applied Mechanics, 48, 2

2. Badel A., Sebald G., Guyomar D., Lallart M., Lefeuvre E., Richard C., 2006, Piezoelectric vibration control by synchronized switching on adaptive voltage sources: Towards wideband semi-active damping, Journal of Acoustics Society American, 119, 5, 2815-2825 
3. Ciminello M., Calabrò A., Ameduri S., Concilio S., 2008, Synchronized Switched Shunt Control technique applied on a cantilevered beam: experimental investigations, Journal of Intelligent Material Systems and Structures, 19, 9, 1089-1100

4. Ciminello M., Lecce L., Ameduri S., Calabrò A., Concilio S., 2010, Multi-tone Switching Shunt Control by a PZT network embedded into a fiberglass panel: design, manufacture, and test, Journal of Intelligent Material Systems and Structures, 21, 437-451

5. Clark W.W., 1999, Semi-active vibration control with piezoelectric materials as variable stiffness actuators, AIAA/ASME/ASCE/AHS/ASC Structures, Structural Dynamics, and Materials Conference and Exhibit, Part 4

6. Guyomar D., Richard C., Petit L., 2001, Non-linear system for vibration damping, 142th Meeting of Acoustical Society of America, Fort Lauderdale, USA

7. Hollkamp J.J., 1994, Multimodal passive vibration suppression with piezoelectric materials and resonant shunts, Journal of Intelligent Material Systems and Structures, 5, 49-56

8. Magliacano D., Viscardi M., Ciminello M., Dimino I., Concilio A., 2016a, Feasibility study for a tonal vibration control system of a mounting bracket for automotive gearboxes, International Journal of Mechanics, 10, 403-410

9. Magliacano D., Viscardi M., Dimino I., Concilio A., 2016b, Active vibration control by piezoceramic actuators of a car floor panel, 23rd International Congress on Sound and Vibration, $I C S V$

10. Makihara K., Onoda J., Minesugi K., 2005, Low-energy-consumption hybrid vibration suppression based on an energy-recycling approach, AIAA Journal, 43, 8, 1706-1715

11. Makihara K., Onoda J., Minesugi K., 2007a, A self-sensing method for switching vibration suppression with a piezoelectric actuator, Smart Materials and Structures, 162, 2, 455-461

12. Makihara K., Onoda J., Minesugi K., 2007b, Comprehensive assessment of semi-active vibration suppression including energy analysis, Journal of Vibration and Acoustics, 129, 84-93

13. Makihara K., Onoda J., Minesugi K., 2007c, Using tuned electrical resonance to enhance bang-bang vibration control, AIAA Journal, 45, 2, 497-504

14. Onoda J., Makihara K., Minesugi K., 2003, Energy-recycling semi-active method for vibration suppression with piezoelectric transducers, AIAA Journal, 41, 4, 711-719

15. Petit L., Lefeuvre E., Richard C., Guyomar D., 2004, A broadband semipassive piezoelectric technique for structural damping, SPIE International Symposium on Smart Structures and Materials: Damping and Isolation, San Diego, CA, USA

16. QIU J., Ji H., ZU K., 2009, Semi-active vibration control using piezoelectric actuators in smart structures, Frontiers of Mechanical Engineering in China, 4, 3, 242-251

17. Richard C., Guyomar D., Audigier D., Ching G., 1998, Semi-passive damping using continuous switching of a piezoelectric device, SPIE Smart Structures and Materials Conference: Passive Damping and Isolation, San Diego

18. Siano D., Viscardi M., Panza M.A., 2016, Automotive Materials: An Experimental Investigation of an Engine Bay Acoustic Performances, Energy Procedia, 101, 598-605

19. Viscardi M., Di Leo R., 2016, Implementation of an electronic circuit for SSSA control approach of a plate type element and experimental match with a feed-forward approach, Archive of Mechanical Engineering, LXIII, 4, 665-677 